\title{
Autonomie als Bezugspunkt einer universalen Medizinethik
}

\author{
Claudia Wiesemann
}

Online publiziert: 19. Oktober 2012

(C) Die Autor(en) 2012. Dieser Artikel ist auf Springerlink.com mit Open Access verfügbar.

Zusammenfassung Das ethische Prinzip des Respekts vor der Autonomie des Patienten/ Probanden hat in der modernen Medizin mittlerweile weltweit Bedeutung erlangt. Die Betonung der Autonomie des Patienten und Probanden in allen in der letzten Zeit verabschiedeten internationalen Deklarationen gibt dieser Tendenz unmissverständlich Ausdruck. Doch wenngleich diese Entwicklung unstrittig positiv ist, wirft sie dennoch eine Reihe von Fragen auf, die mit der Kodifizierung, Interpretation, Reichweite und Anwendung dieses universalen Prinzips verbunden sind. Die Antworten auf diese Fragen entscheiden darüber, ob Autonomie als hilfreiches, emanzipatorisches oder als überforderndes, gar hegemoniales Prinzip begriffen wird. Diese normativen Probleme werden anhand von Beispielen aus der globalen Medizinethik verdeutlicht. Was in modernen Gesellschaften als Autonomie bezeichnet und für die Praxis fruchtbar gemacht wird, ist als ein soziales Konstrukt zu verstehen, nicht im Sinne einer Schimäre, sondern im Sinne einer soziokulturellen Leistung, die auf komplexen, historisch gewachsenen und kulturell geprägten Interaktionen von Gesellschaften, Institutionen und Individuen beruht.

Schlüsselwörter Autonomie · Universale Medizinethik · Patient · Globalisierung · Informierte Einwilligung

\section{Autonomy as a point of reference for universal medical ethics}

Abstract Definition of the problem In modern medical science, the ethical principle of respect for patient/proband autonomy has gained wide recognition. All international declarations which have been passed more recently clearly emphasize the patient's and test subject's autonomy. While this is an undeniably positive development, it also raises a

Prof. Dr. med. C. Wiesemann $(\bowtie)$

Abteilung Ethik und Geschichte der Medizin, Universität Göttingen,

Humboldtallee 36, 37073 Göttingen, Deutschland

E-Mail: cwiesem@gwdg.de 
number of questions. The answer to these questions determines whether autonomy should be regarded as a helpful, emancipatory or an overburdening, even hegemonic, principle. Arguments By drawing on examples from global medical ethics, the paper discusses the validity, interpretation, scope, and implementation of the principle of respect for autonomy. Conclusion Autonomy in modern societies has to be conceived as a social construct, not in the sense of a chimaera but as a sociocultural achievement. It relies on complex, historically contingent, and culturally determined interactions of societies, institutions, and individuals.

Keywords Autonomy · Universal medical ethics · Patient · Globalization · Informed consent

\section{Respekt vor Autonomie und die moderne Medizin}

2005 verabschiedete die Organisation der Vereinten Nationen für Erziehung, Wissenschaft und Kultur (UNESCO) eine Universal Declaration on Bioethics and Human Rights. In Art. 5 heißt es dort:

The autonomy of persons to make decisions, while taking responsibility for those decisions and respecting the autonomy of others, is to be respected [27].

Wie dieses Beispiel zeigt, hat das ethische Prinzip der Autonomie in der Medizin mittlerweile weltweit Bedeutung erlangt. Die Betonung der Autonomie des Patienten und Probanden in allen in der letzten Zeit verabschiedeten internationalen Deklarationen gibt dieser Tendenz unmissverständlich Ausdruck. ${ }^{1}$ Es ist die Antwort auf andere, nicht weniger globale gesellschaftliche Phänomene: einer weltweiten Verbreitung der wissenschaftlich-technisch geprägten Medizin, weltweit miteinander vernetzter scientific communities, universaler Wissenskorpora, global agierender Medizinindustrie-Unternehmen sowie einer weltweiten Verbreitung sozialer Institutionen und Systeme der Gesundheitsvorsorge wie dem Krankenhaus oder der Krankenversicherung. Alle diese recht heterogenen Entwicklungen gleichen sich in einer Hinsicht: Sie mindern die Bedeutung der individuellen Beziehung von Patient und Arzt, indem der Patient (oder Proband) in der Person des Arztes nicht mehr nur einer für ihn zuständigen und ihm verpflichteten Person begegnet, sondern auch einem Vertreter des Gesundheitswesens und seiner Institutionen, einem Inhaber von Herrschaftswissen in Form wissenschaftlicher Deutungen des Patientenbefindens und eines Agenten eines sich gelegentlich verselbständigenden technologischen Imperativs [14, 17, 28].

Die Stärkung der Person des Patienten oder Probanden durch das ethische Prinzip der Autonomie muss als eine notwendige und sinnvolle Maßnahme gegen diese Entwicklungen verstanden werden. Das von einigen Medizinethikern gezeichnete Zerrbild des medizini-

\footnotetext{
${ }^{1}$ Einige Beispiele für Übereinkommen auf europäischer oder internationaler Ebene: Übereinkommen zum Schutz der Menschenrechte und der Menschenwürde im Hinblick auf die Anwendung von Biologie und Medizin: Übereinkommen über Menschenrechte und Biomedizin (http://conventions.coe.int/Treaty/Commun/QueVoulezVous.asp?NT=164\&CM=1\&CL=GER); Zusatzprotokoll zum Übereinkommen über Menschenrechte und Biomedizin betreffend biomedizinische Forschung (http://conventions.coe.int/Treaty/ Commun/QueVoulezVous.asp?CL=GER\&NT=195); Charta der Grundrechte der Europäischen Union (http://www.europarl.europa.eu/charter/pdf/text_de.pdf); Deklaration des Weltärztebundes von Helsinki (http://www.wma.net/en/30publications/10policies/b3/index.html); CIOMS Guidelines (http://www.cioms. ch/publications/layout_guide2002.pdf); Universal Declaration on the Human Genome and Human Rights (www.unesco.org/new/en/social-and-human-sciences/themes/bioethics/human-genome-and-human-rights/) (Alle Quellen zugegriffen: 22. Nov. 2011).
} 
schen Paternalismus früherer Zeiten ist zunächst einmal eine in die Vergangenheit projizierte Kritik an einer solcherart scheinbar uferlosen und nicht durch individuelle Rechte ausbalancierten medizinisch-technisch-industriellen Entwicklung. Doch auch ohne eine solche der Moderne geschuldete Potenzierung ihrer Macht läuft die Medizin qua gesellschaftlichen Ranges Gefahr, Angehörige benachteiligter oder gar marginalisierter Gruppen nicht ausreichend zu respektieren. Arme Kranke, Frauen, Kinder, psychisch Kranke oder Anstaltsinsassen konnten ihre Interessen in medizinischen Entscheidungen oft nicht gegen gesellschaftlich mächtigere Instanzen - nicht nur den Arzt, sondern auch die eigene Familie oder die Anstaltsleitung - durchsetzen [28]. Überlegenes körpertechnisches Wissen der Ärzte und die große soziale Bedeutung von Gesundheit können jedoch als Gründe nicht ausreichen, Patienten die Anerkennung als Personen zu verweigern. Es scheint heute fast überflüssig, dies anzumerken.

Doch den Vertretern des Prinzips der Autonomie in der Medizin wird gelegentlich der Einwand entgegengehalten, sie befürworteten eine hegemoniale Dominanz westlicher Werte gegenüber anderen Kulturen. Die Betonung der Patienten-/Probandenautonomie in der nicht-westlichen Welt wird dabei als moderne Form des Kolonialismus oder Imperialismus karikiert, als Überstülpung westlicher Lebensart mit den subversiven Mitteln einer vermeintlich neutralen Wissenschaftsethik. Dem wird z. B. in den asiatischen Ländern ein familism entgegengehalten, also die Überzeugung, dass gemeinschaftliche, zumeist familiäre Werte zu Recht einen höheren Rang als die Autonomie des Individuums beanspruchen [12]. Aber auch in der westlichen Welt regt sich Kritik gegen eine moralisch einseitige Ausrichtung auf individuelle Wahlfreiheit, wenn sich diese vermeintliche Wahlfreiheit vor dem Hintergrund impliziter gesellschaftlicher Zwänge vollzieht und diese zu kaschieren droht, wie bei der pränatalen Diagnostik, oder wenn sie sich in Gesellschaften mit mangelhaftem öffentlichen Gesundheitswesen überhaupt nur den Wohlhabenden bietet [1,16]. Diese Einwände - selbst die fundamentale Kritik von Seiten der vorgeblich konfuzianischen Asian values - gehen jedoch nicht so weit, die normative Bedeutung personaler Autonomie grundsätzlich in Zweifel zu ziehen. Es geht vielmehr um Fragen der Einordnung dieses Prinzips in einen größeren moralischen, gesellschaftlichen und politischen Kontext, um ihm - durchaus in kritischer Auseinandersetzung - eine Tiefendimension zu verleihen.

In diesem Sinne soll hier gefragt werden, wie man sinnvoller Weise von einer universalen Gültigkeit des Prinzips der Autonomie in der Medizin sprechen kann. Die Auseinandersetzungen um das Konzept der Autonomie des Patienten/Probanden haben wegen der Allgegenwart der zugrunde liegenden Modernisierungsprozesse wohl mehr als alles andere zur weltweiten Konjunktur des Fachs Medizinethik bzw. Bioethik beigetragen. Patienten-/ Probandenautonomie ist unzweifelhaft jenes ethische Prinzip, das als gemeinsame konzeptuelle Grundlage der Arbeit von Medizin- und Bioethiker(inne)n weltweit dient. In dieser deskriptiven Hinsicht kann man von einem Prinzip einer universalen Medizinethik sprechen. Letztlich hat die Medizinethik in der Moderne selbst ihren besten Verbündeten. Denn moderne Gesellschaften setzen nicht nur auf Verwissenschaftlichung und Technik, sie fördern auch die Freisetzung des Individuums aus Traditionen und gesellschaftlichen Zwängen und befördern damit wesentlich die Herausbildung der Idee einer moralischen Instanz in Gestalt der selbstbestimmt entscheidenden Person, die das Gleichgewicht der Kräfte in der Medizin wiederherstellen soll.

Doch wenngleich diese Entwicklung unstrittig positiv ist, wirft sie auch eine Reihe von Fragen in normativer Hinsicht auf, die mit der Geltung, Interpretation, Reichweite und Anwendung dieses universalen Prinzips verbunden sind. Die Antworten auf diese Fragen entscheiden letztlich darüber, ob Autonomie als hilfreiches, emanzipatorisches oder als überforderndes, gar hegemoniales Prinzip begriffen werden kann oder muss. Auf sie soll 
im Folgenden näher eingegangen werden. Ziel dieses Beitrags ist es mithin, Facetten der Geltung, Interpretation, Reichweite und Anwendung dieses Prinzips anhand von Beispielen aus unterschiedlichen globalen Kontexten zu beschreiben, um daran die Problematik einer universalen normativen Bedeutung zu verdeutlichen.

\section{Kodifizierung von Autonomie}

Weltweit gibt es keinen einheitlichen Weg, der Autonomie als moralischem Prinzip in modernen Gesellschaften mit den Mitteln des Rechts Geltung zu verschaffen. Die Autonomie des Patienten/Probanden wird in demokratisch verfassten Gesellschaften zwar nicht selten in rechtlicher Form kodifiziert, dies jedoch nicht nur modifiziert nach unterschiedlichen Rechtstraditionen, sondern auch nach regionalen kulturellen Besonderheiten. In den USA ist bspw. seit 1990 das Patient Self-Determination Act (PSDA) in Kraft, das Patienten sehr weitgehende Selbstbestimmungsrechte einräumt; es hat jedoch nur Auswirkungen auf durch die Öffentliche Hand finanzierte Einrichtungen. Gleiches gilt für die schon in den siebziger Jahren verabschiedete, ausführliche Kodifizierung der Forschung am Menschen, welche die Rechte des Probanden auf Selbstbestimmung sichert, dies jedoch nur für vom Staat geförderte Forschungsprojekte. Privat finanzierte Forschung ist bis heute - einer besonderen rechtskulturellen Logik folgend, die dem Staat keine so allgemeine Wächterfunktion zugesteht, wie dies bspw. in Deutschland üblich ist - rechtlich ungeregelt. In den USA findet sich deshalb z. B. in privaten Gefängnissen ein breites Spektrum medizinischer Forschung, bei der die Rechte der Versuchsteilnehmer nicht einheitlich geregelt sind und die Anwendung ethischer Prinzipien weitgehend lokalen Initiativen und lokaler Kontrolle überlassen ist. Eine staatliche Aufsicht darüber gibt es nicht [15].

International unterschiedlich interpretiert wird nicht nur die Notwendigkeit einer rechtlichen Verankerung des Autonomieprinzips in Forschung oder Klinik, sondern auch die Art und Weise, wie dabei zwischen individuellen und Gruppeninteressen zu vermitteln ist. Taiwan bspw. hat das Recht des Patienten auf umfassende Information kodifiziert; da die Familie jedoch ebenso eine starke Rechtsposition genießt, wird ihr generell eine Möglichkeit zur Mitbestimmung eingeräumt. Im Physician's Act von 2002 heißt es deshalb in Art. 12 Abs. 1:

When diagnosing and treating patients, a physician shall inform the patient or the patient's family of the status of the disease, treatment principles, treatment, medication, prognosis and possible unfavorable reactions (vgl. [8], S. 145).

Gewollt ist eine Balance von individueller Zustimmung durch den Patienten und einer Beteiligung der Familie. Ähnliche Lösungen, die zwischen Individuum und Familie eine Vermittlung anstreben, finden sich im deutschen Recht, z. B. bei der Regelung der postmortalen Organentnahme. Braune et al. identifizieren in einem Vergleich verschiedener ostasiatischer Länder und Deutschland unterschiedliche Modelle im Hinblick auf die Art und Weise, wie Individual- und Gruppeninteressen gegeneinander ausbalanciert werden:

Der Übergang vom Individual Informed Consent zum Family Consent hat viele Nuancen. Entsprechend haben sich unter diesem begrifflichen Schirm Differenzierungen wie Family Decision-Making, Family Consultation oder Family Involvement entwickelt ([8], S. 147).

Dabei werden der Familie in unterschiedlichem Maße Mitspracherechte eingeräumt. Während dieses Vorgehen aus der Perspektive eines individualistischen Ansatzes die Bedeutung 
des Prinzips Autonomie substantiell schmälerte, kann aus der Sicht des familism nur eine solche Unterstützung durch die Familie echte Autonomie möglich machen.

\section{Interpretation von Autonomie}

Die Interpretation des Prinzips der Autonomie ist in einem nicht unbeträchtlichen Maße von dem dahinter stehenden Menschenbild abhängig. Als Persönlichkeitsideal bzw. Fähigkeit von Personen wird das Verständnis von Autonomie in hohem Maße von kulturellen Mustern situations- und rollenspezifisch geprägt. Je nachdem, ob z. B. die autonome Person als ein von Mitmenschen „losgelöster, entkörperlichter, verallgemeinerter Anderer“ oder als ein auf Beziehungen angewiesenes, sozial eingebettetes, umweltbezogenes Wesen verstanden wird ([5], S. 177), werden auch die notwendigen Bedingungen autonomen Handelns unterschiedlich aufgefasst. Beauchamp und Childress setzen z. B. in ihrem Konzept von Handlungsautonomie einen ,normal chooser" voraus, um zu hohe Ansprüche an Selbstbestimmung im Handeln zu vermeiden. Ein normal chooser ist nach ihren eigenen Beispielen jemand, der eine finanzielle Investition tätigt, einen Mitarbeiter einstellt oder ein neues Haus kauft ([3], S. $101 \mathrm{f}$.). Schon an diesen Beispielen wird allerdings deutlich, dass eine solche Auffassung von menschlicher Normalität nicht universell gültig sein kann, schildern sie doch typische Situationen von Personen der westlichen Mittelschicht, in denen i.d. R. ohne tiefere emotionale Beteiligung und ohne existentielle Betroffenheit (der entscheidenden Person selbst oder einer anderen, ebenfalls betroffenen) Beschlüsse gefällt werden. Es ist aber strittig, obman bei der Entscheidung, Leihmutter zu werden oder einem Angehörigen ein Organ zu spenden, von solchen Normalitäts-Vorstellungen ausgehen sollte.

Beauchamp und Childress diskutieren selbst am Beispiel der Navajo-Indianer ein in ihren Augen auf das zugrunde liegende Menschenbild zurückzuführendes Dilemma. Navajo-Indianer vertreten eine besondere, kulturell verankerte Auffassung des menschlichen Geistes als einem von seiner Umwelt nicht losgelösten und loslösbaren Vermögen. Im traditionellen Denken dieses Stammes beinhaltet allein das Reden über etwas die Möglichkeit, dieses Etwas hervorzubringen ([3], S. 106). Eine Aufklärung über die Nebenwirkung einer Krankheit oder einer Behandlung kann demnach ausgerechnet diese negativen Konsequenzen zur Folge haben. Unter welchen Umständen kann dann aber die Verweigerung eines Patienten, informiert zu werden, oder gar seine Zustimmung zu einer Maßnahme noch als autonom eingestuft werden? Anders ausgedrückt: Kann man unter einer autonomen Entscheidung auch die Entscheidung gegen die Voraussetzungen von Autonomie fassen, oder ist es in diesem Fall überhaupt nicht mehr sinnvoll, von Autonomie auszugehen?

Das Menschenbild spielt auch dann eine gewichtige Rolle, wenn man Autonomie nicht rein prozedural als „Wille zu wollen“2, sondern als substantiell gehaltvoll, als wahrhaft freie Entscheidung, die den eigenen lebensgeschichtlich begründeten Interessen entspricht, versteht $[9,19]$. Dann nämlich muss sich jede Gesellschaft fragen, was sie unter Freiheit, echten eigenen Interessen oder Authentizität versteht. ${ }^{3}$ Wird z. B. die Entscheidung, sich selbst zu töten, überhaupt als der Möglichkeit nach frei aufgefasst? Gegen welche eigenen Interessen darf ein Individuum verstoßen, ohne seinen Anspruch auf Autonomie zu untergraben, mithin als ernsthaft psychisch krank zu gelten? Prozedurale - und damit inhaltsneutrale - Definitionen von Autonomie würden es zwar erlauben, auf kulturell bedingten

\footnotetext{
${ }^{2}$ Für einen Überblick s. [10, 24]. Zur Kritik an Frankfurts einflussreichem prozeduralen Ansatz s. [6, 22].

${ }^{3}$ Für einen beispielhaften Vergleich der Bedeutung von Freiheit und eigenen Interessen bei Entscheidungen am Lebensende in Deutschland und Israel s. [25].
} 
Vorannahmen über das Wesen des Menschseins zu verzichten. Sie sind aber gerade in der Medizin, die oft mit den dramatischen Auswirkungen des menschlichen Körpers auf geistige Prozesse konfrontiert ist, von eingeschränktem Nutzen. Der Heroinabhängige z. B. will eben nicht nur den „Schuss“, er findet für dieses Wollen auch eine Vielzahl sekundärer, vermeintlich rationaler Begründungen, die u. U. ohne Bezug auf eine substantiell gehaltvolle Vorstellung von Freiheit nicht als solche zu entlarven sind. ${ }^{4}$

\section{Reichweite von Autonomie}

Fragen nach der Reichweite des Autonomiekonzepts können in zweierlei Hinsicht in globaler Perspektive Bedeutung erlangen. Zum einen geht es um das Problem, welchen Menschen Autonomie zugesprochen wird. Gehören dazu z. B. auch Jugendliche oder gar Kinder? Die Antwort auf diese Frage bemisst sich üblicherweise neben der rechtlichen Grenze der Volljährigkeit am Kriterium der Kompetenz, also dem Vermögen des Individuums, Wesen, Tragweite und Bedeutung einer medizinischen Maßnahme zu verstehen und seinen Willen danach zu bestimmen. Kompetenz erhält damit eine sogenannte ,gatekeeper function“ ([3], S.111). Doch mit diesem Maßstab ist noch nicht viel gewonnen, muss doch zugleich entschieden werden, ob Wesen, Tragweite und Bedeutung aus der Perspektive des Kindes bzw. Jugendlichen oder aus der Perspektive der beteiligten Erwachsenen den Ausschlag geben sollen. Die dreizehnjährige, leidgeprüfte junge Patientin mit chronischer Leukämie, die sich, weil sie keine Ärzte und Krankenhäuser mehr sehen will und Sehnsucht nach ihrem Zuhause hat, gegen eine lebensrettende Herztransplantation entscheidet, nimmt die Bedeutung dieser Entscheidung ganz aus ihrer kindlichen Lebenswelt wahr und ohne die ihr im Falle der Einwilligung in die Therapie eventuell noch möglichen Jahre eines erwachsenen Lebens ernsthaft in Betracht ziehen zu können. Wenn man ihr dennoch, wie in England geschehen, Entscheidungsautonomie konzediert, dann wertet man die subjektive Perspektive des Kindes als hinreichend für Kompetenz. ${ }^{5}$ In den USA dagegen konzediert die Mehrheit der Bundesstaaten grundsätzlich erst den über 18-Jährigen Autonomie in Gesundheitsfragen [21]. Über die Wertigkeit solcher Grenzfälle wird somit nicht zuletzt aufgrund sozialer und kultureller Unterschiede weltweit unterschiedlich entschieden, dies ist unter anderem abhängig davon, in welchem Maßstab die betroffenen Personengruppen - neben Kindern und Jugendlichen gehören dazu auch geistig behinderte oder aber demenzkranke Personen - auch sonst gesellschaftliche Förderung und Unterstützung bei der eigenständigen Wahrung ihrer Interessen erfahren.

Darüber hinaus stellt sich zum anderen aber auch die Frage, welche Optionen überhaupt als dem Bereich autonomer Entscheidung zugehörig betrachtet werden. Mit seinem über dreißig Jahre, bis 1997, andauernden Verbot einer postmortalen Organentnahme für Transplantationszwecke hat Japan lange Zeit demonstriert, dass eine derartige Verfügung über den eigenen Körper nicht dem Bereich autonomer Entscheidungen zugerechnet werden sollte [18]. Auch heute noch koppeln viele Länder dieser Welt, unter ihnen Deutschland, das Recht auf Spende eines Organs zumindest an das Vorliegen des Hirntods, selbst wenn unter anderen Umständen der Tod aufgrund einer schweren Erkrankung vorhergesehen eintritt (d.h. bei den sog. non-heart-beating donors). Große Diskrepanzen weltweit lasse sich auch bei der Antwort auf die Frage konstatieren, ob es der Entscheidung des Individuums überlassen sein soll, wesentliche Teile des Körpers, z. B. eine Niere, zu spenden oder zu ver-

\footnotetext{
${ }^{4}$ Für eine weitergehende Diskussion s. [26].

${ }^{5}$ Für die journalistische Darstellung des Falls s. [2].
} 
kaufen. Ähnlich verlaufen Diskussionen im Bereich der Fortpflanzung. In Deutschland ist derzeit Samenspende erlaubt, Eizellspende aber nicht. In Indien ist die kommerzielle Leihmutterschaft der individuellen Entscheidung der beteiligten Frau(en) überlassen, in Israel ebenso, jedoch unter gewissen staatlichen Auflagen, in den USA wird dies von Bundesland zu Bundesland unterschiedlich geregelt. Neben Überlegungen, welche im Falle von Fortpflanzung v. a. das Wohlergehen und die Identität des Kindes betreffen, geht es hierbei um die Frage, ob das Konzept der Autonomie die Möglichkeit zur Verdinglichung des eigenen Körpers und insbesondere zur Kommerzialisierung von wesentlichen Körpersubstanzen oder -funktionen erfasst und erfassen soll.

\section{Anwendung von Autonomie}

Schließlich lässt sich das Prinzip der Autonomie auch im Einzelfall nicht anwenden, ohne damit Fragen seiner allgemeinen Bedeutung und seiner Gewichtung im Vergleich zu anderen moralischen Werten aufzuwerfen. Über eine gelingende Integration von Autonomie und Gemeinschaft herrschen, so die Sozialpsychologin Joan G. Miller, abhängig von der Kultur, in der man lebt, ganz allgemein unterschiedliche Idealvorstellungen ([20], S. 359). Darüber hinaus werden in vielen Ländern Situationen, in denen sich Wahlmöglichkeiten eröffnen könnten, schon vorgängig durch gesellschaftliche Entscheidungen eingeschränkt. Dies gilt besonders für Gesundheits- und Krankheitsleistungen. Alle Länder mit einem nationalen Gesundheitswesen grenzen aus ökonomischen Gründen das Angebot medizinischer Leistungen ein und beschränken so die freie Wahl des Patienten im Vorhinein, oft sogar ohne dessen Wissen. Wenngleich dies zumeist aus dem guten Grund einer beschränkten Wirksamkeit dieser Angebote geschieht, sind damit doch stets auch verallgemeinerbare Wertentscheidungen aus gesellschaftlicher Perspektive (z. B. im Hinblick auf Wirtschaftlichkeit und Kostengünstigkeit) verbunden, die der Vorstellung von Autonomie als Verwirklichung je eigener Wertvorstellungen zuwider laufen. Hier wird Autonomie gegen die moralischen Prinzipien der Gerechtigkeit, Solidarität oder Benefizienz abgewogen, zumeist ohne die Betroffenen daran direkt zu beteiligen [29].

Umgekehrt besteht in Ländern ohne ein nationales Gesundheitswesen die auf den ersten Blick größere Wahlfreiheit oft nur auf dem Papier, da nur die Wohlhabenden bzw. jene, die sich die besseren Versicherungen leisten können, von solcher Wahlfreiheit überhaupt Gebrauch machen können. Seit der Zerschlagung des alten, noch unter kommunistischer Herrschaft eingerichteten allgemeinen Gesundheitswesens hat sich in China eine solche Situation vermeintlicher Wahlfreiheit etabliert. Dies hat u. a. zur Folge, dass die Rolle der Familie gegenüber der autonomen Entscheidung des Patienten wieder aufgewertet wurde. Ärzte verlangen nun oft neben der Unterschrift des Patienten unter das Aufklärungsformular eine zweite seitens eines Familienmitglieds (second signature). Diese soll sicherstellen, dass die Familie auch nach dem ggf. eintretenden Tod des Patienten die Kosten der Behandlung übernimmt [8].

An solchen Beispielen zeigt sich nicht nur, dass sich der Rang des Prinzips der Autonomie im konkreten Einzelfall erst im Vergleich zu anderen moralischen Prinzipien erweist, es wird auch offensichtlich, dass mit diesem Abgleich der Gehalt des Prinzips selbst modifiziert wird. So lässt sich auf der einen Seite ein Prinzip der absoluten Wahlfreiheit denken, das durch keinerlei gesellschaftlich-normative Vorentscheidungen eingeengt werden darf, weil eine derartige normative Einigung in pluralistischen Gesellschaften als nicht möglich 
angesehen wird. ${ }^{6}$ Auf der anderen Seite sind - ebenfalls innerhalb des Kreises liberal verfasster Gesellschaften - gleichermaßen Auffassungen des Prinzips der Autonomie denkbar, die auf einer gesellschaftlichen Einigung über gemeinsame, intersubjektiv geteilte Standards rationalen Entscheidens beruhen und damit den Gehalt autonomen Entscheidens von vornherein in einem gesellschaftlich verträglichen Maß begrenzen [23].

Sollten sich die Erwartungen, die an die moderne Gentechnologie gerichtet werden, bewahrheiten, sollten also in nicht allzu ferner Zukunft tatsächlich mit den Mitteln der Lebenswissenschaften menschliche Eigenschaften gezielt durch genetische Eingriffe ausgewählt werden können, wird das Prinzip der Autonomie, und damit die Grundlage liberaler Gesellschaften, so die Diagnose der Politikwissenschaftlerin und Medizinethikerin Katharina Beier, noch um einiges mehr unter Druck geraten [4].

\section{Schlussfolgerungen}

In welcher Weise das Prinzip der Autonomie für eine universale Medizinethik Bedeutung beanspruchen kann, diese Frage lässt sich nicht allein durch eine theoretische, normative Analyse beantworten, sie bedarf vielmehr gleichermaßen einer Analyse des faktischen Einsatzes im globalen Kontext. Betrachtet man die Kodifizierung, Interpretation, Reichweite und Anwendung vor dem Hintergrund verschiedener medizinischer, kultureller und politischer Systeme, wird diesem Prinzip die notwendige Tiefendimension verliehen, ohne die jede Rede von dessen universaler Bedeutung abstrakt und inhaltsleer bleiben muss. Diese Systeme nehmen Einfluss auf Wahrnehmung, Gewichtung und praktische Bedeutung von Autonomie als moralischem Recht, als Persönlichkeitseigenschaft, als Fähigkeit von Personen oder als situativer Disposition [7, 13]. Die Ausdeutung dieses so bedeutsamen ethischen Prinzips erfolgt stets in Wechselwirkung mit gesellschaftlichen und kulturellen Faktoren.

Was als Autonomie bezeichnet wird, sei es als theoretisches Konzept, sei es als faktisch für notwendig gehaltener und gewährter Freiraum individuell autonomen Handelns, ist in modernen, demokratisch verfassten Gesellschaften als ein soziales Konstrukt zu verstehen, nicht im Sinne einer Schimäre, sondern im Sinne einer soziokulturellen Leistung, die auf komplexen, historisch gewachsenen und kulturell geprägten Interaktionen von Gesellschaften, Institutionen und Individuen beruht. Dies schränkt die Bedeutung von Autonomie in der Medizinethik nicht ein. Es lässt die Auseinandersetzung mit ihr nur als umso wichtiger erscheinen.

Danksagung Die Arbeit zu diesem Aufsatz wurde unterstützt von der VolkswagenStiftung im Rahmen der Forschergruppe an der Universität Göttingen „Autonomie und Vertrauen in der modernen Medizin. Erkenntnis - Praxis - Norm“ (www.autonomie-und-vertrauen.uni-goettingen.de/). Ich danke Sabina Fazli für die Übersetzung von Titel und Abstract.

Interessenkonflikt Die Autorin gibt an, dass keine Interessenkonflikte bestehen.

Open Access Dieser Artikel unterliegt den Bedingungen der Creative Commons Attribution License. Dadurch sind die Nutzung, Verteilung, und Reproduktion erlaubt, sofern der/die Originalautor/en und die Quelle angegeben sind.

\section{Literatur}

1. Azevedo ES (2002) Decisions in circumstances of poverty. Eubios J Asian Int Bioeth 12:105-107

2. BBC (2008) Girl wins right to refuse heart. BBC News (11.11.2008) http://news.bbc.co.uk/2/hi/uk news/england/hereford/worcs/7721231.stm. Zugegriffen: 23. Nov. 2011

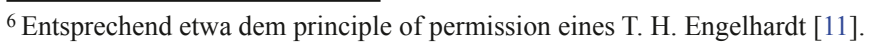


3. Beauchamp T, Childress J (2009) Principles of biomedical ethics, 6. Aufl. Oxford University Press, New York

4. Beier K (2009) Zwischen Beharren und Umdenken. Die Herausforderung des politischen Liberalismus durch die moderne Biomedizin. Campus, Frankfurt a. M.

5. Benhabib S (1995) Selbst im Kontext. Kommunikative Ethik im Spannungsfeld von Feminismus, Kommunitarismus und Postmoderne. Suhrkamp, Frankfurt a. M.

6. Betzler M (2001) Bedingungen personaler Autonomie. In: Betzler M, Guckes B (Hrsg) Harry G. Frankfurt. Freiheit und Selbstbestimmung. Akademie Verlag, Berlin, S 17-49

7. Birnbacher D (1997) Patientenautonomie und ärztliche Ethik am Beispiel der prädiktiven Diagnostik. Jahr Wiss Ethik 2:105-118

8. Braune F, Wiesemann C, Biller-Andorno N (2008) Informed Consent und seine Konkretisierung in der internationalen Bioethik: Zur medizinethischen Bedeutung von Aufklärung und Zustimmung in Taiwan und Deutschland. In: Biller-Andorno N, Schaber P, Schulz-Baldes A (Hrsg) Gibt es eine universale Bioethik? Mentis, Zürich, S 135-158

9. Christman J (1989) Introduction. In: Christman J (Hrsg) The inner citadel. Essays on individual autonomy. Oxford University Press, Oxford, S 3-23

10. Christman J (2011) The politics of persons. Individual autonomy and socio-historical selves. Cambridge University Press, New York

11. Engelhardt TH (1996) The foundations of bioethics, 2. Aufl. Oxford University Press, New York

12. Fan R (1998) Critical care ethics in Asia: global or local? J Med Philos 23:549-562

13. Feinberg J (1989) Autonomy. In: Christman J (Hrsg) The inner citadel. Essays on individual autonomy. Oxford University Press, Oxford, S 27-53

14. Göckenjan G (1985) Kurieren und Staat machen. Gesundheit und Medizin in der bürgerlichen Welt. Suhrkamp, Frankfurt a. M.

15. Gostin LO (2007) Biomedical research involving prisoners: ethical values and legal regulation. JAMA 7:737-740

16. Haker H (2002) Ethik der genetischen Diagnostik. Mentis, Paderborn

17. Jütte R (1996) Vom Hospital zum Krankenhaus: 16.-19. Jh. In: Labisch A, Spree R (Hrsg) „Einem jeden Kranken in einem Hospitale sein eigenes Bett“. Zur Sozialgeschichte des Allgemeinen Krankenhauses in Deutschland im 19. Jh. Campus, Frankfurt a. M., S31-50

18. Lock M (2001) Organ transplantation and the invention of death. University of California Press, Berkeley

19. Mackenzie C, Stoljar N (2000) Introduction: autonomy refigured. In: Mackenzie C, Stoljar N (Hrsg) Relational autonomy. Feminist perspectives on autonomy, agency, and the social self. Oxford University Press, New York, S 3-34

20. Miller JG (2000) Verträgt sich Gemeinschaft mit Autonomie? Kulturelle Ideale und empirische Wirklichkeiten. In: Edelstein W, Nunner-Winkler G (Hrsg) Moral im sozialen Kontext. Suhrkamp, Frankfurt a. M., S 337-362

21. Piker A (2011) Balancing liberation and protection: a moderate approach to adolescent health care decision-making. Bioethics 25:202-208

22. Quante M (2000) The things we do for love. Zur Weiterentwicklung von Frankfurts Analyse personaler Autonomie. In: Betzler M, Guckes B (Hrsg) Autonomes Handeln. Beiträge zur Philosophie von Harry G. Frankfurt. Akademie Verlag, Berlin, S 117-136

23. Quante M (2010) Menschenwürde und personale Autonomie. Demokratische Werte im Kontext der Lebenswissenschaften. Meiner, Hamburg

24. Rössler B (2011) Autonomie. In: Stoecker R, Neuhäuser C, Raters ML (Hrsg) Handbuch Angewandte Ethik. Metzler, Stuttgart, S 93-99

25. Schicktanz S, Raz A, Shalev C (2010) The cultural context of patient autonomy and doctors duties: passive euthanasia and advance directives in Germany and Israel. Med Health Care Philos 4:363-369

26. Taylor JS (2005) Introduction. In: Taylor JS (Hrsg) Personal autonomy. New essays on personal autonomy and its role in contemporary moral philosophy. Cambridge University Press, Cambridge, S 1-29

27. UNESCO (2005) Universal declaration on bioethics and human rights (adopted by UNESCO's General Conference on 19 October 2005). http://www.unesco.org/new/en/social-and-human-sciences/themes/ bioethics/bioethics-and-human-rights/. Zugegriffen: 22. Nov. 2011

28. Wiesemann C (1997) Das Recht auf Selbstbestimmung und das Arzt-Patient-Verhältnis aus sozialgeschichtlicher Perspektive. In: Toellner R, Wiesing U (Hrsg) Geschichte und Ethik in der Medizin, Medizin-Ethik, Bd. 10. Gustav Fischer, Stuttgart, S 67-90

29. Wiesemann C (2001) Selbstbestimmte Patienten? - Die Nutznießer der Medizin und ihre Rechte. Gesundheitswes 63:591-596 